\title{
Joint probability distributions for optical parametric down-conversion
}

\section{Jan Peřina \\ perina@prfnw.upol.cz \\ Jaromír Křepelka}

\begin{abstract}
Department of Optics, Palacký University, 17. listopadu 50, 77207 Olomouc, Czech Republic. Joint Laboratory of Optics, Pala4cký University and Institute of Physics of Academy of Sciences of the Czech Republic, 17. listopadu 50, 77207 Olomouc, Czech Republic.

Joint Laboratory of Optics, Palacký University and Institute of Physics of Academy of Sciences of the Czech Republic, 17. listopadu 50, 77207 Olomouc, Czech Republic.
\end{abstract}

We derive multi mode joint generating functions, photon-number distributions, integrated-intensity distributions and related quantities for spontaneous and stimulated parametric down-conversion in relation to experiments. [DOI: 10.2971/jeos.2006.06002]

Keywords: Quantum measurement, parametric down-conversion, non classical light

\section{Introduction}

Nonlinear process of optical spontaneous parametric downconversion is a fundamental quantum process useful for verification of various quantum phenomena and for their applications [1]-[3]. The theory of this process has been well worked out, especially when pumping beams are strong. In recent experiments $[4,5]$ (and references therein) joint distributions of photon pairs have been measured or reconstructed from measured experimental data. The corresponding description derived from the first principles was proposed in [6].

In this connection we can mention the earlier experimental and theoretical investigations of quantum statistics of photon pairs from spontaneous parametric down-conversion, as discussed from other points of view, such as detection process [7]-[9], "more intense" beams [10], correlations and quantum interference [11, 12] non classical nature [13], spatial properties $[14,15]$ and polarisation properties $[16,17]$. It is possible to create moiré fringe effects in spatial correlations of twin photons [18], using two down-converters one can transfer quantum correlations [19] and twin photons were used to generate entangled states [20].

Recently various experiments were performed with process of stimulated down-conversion [21]-[25] including optical micro structure fibres [26]. In this paper we briefly review the description of the spontaneous down-conversion in regimes of weak photon beams [6] and of the stimulated downconversion [27] giving multi mode formulae for joint generating function, joint photon-number distribution and joint integrated-intensity probability distribution in signal and idler modes for the multi mode process, provided that the pumping beam is strong and coherent.

\section{Q U A N T U M $S C R I P T I O N$ \\ STATISTICA L DE -}

When using the standard methods of quantum optics based on the coherent-state technique, we can adopt results for the description of two-mode non degenerate optical parametric process with strong classical coherent pumping, as reviewed in [28] (section 10.1.2), including losses and noise. Assuming equivalent pairs of photons arising in the process, the $s$ ordered $2 M$-mode joint generating function can be obtained in the spirit of the Mandel-Rice formula in the form [27]

$$
G_{S}\left(\lambda_{1}, \lambda_{2}\right)=\frac{1}{D_{s}^{M}} \exp \left[-\frac{\lambda_{1}\left|\xi_{1}\right|^{2}+\lambda_{2}\left|\xi_{2}\right|^{2}+\lambda_{1} \lambda_{2} L_{s}}{D_{s}}\right],
$$

where $\lambda_{1}$ and $\lambda_{2}$ are parameters of the generating function, $D_{s}=1+\lambda_{1} B_{1 s}+\lambda_{2} B_{2 s}+\lambda_{1} \lambda_{2} K_{s}, K_{s}=B_{1 s} B_{2 s}-\left|D_{12}\right|^{2}=$ $\left(B_{1}+B_{2}\right)(1-s) / 2 M+K+(1-s)^{2} / 4=\langle n\rangle_{\text {spont }}(1-$ $s) / 2 M+K+(1-s)^{2} / 4$, which is just the determinant of the Fourier transformation $K=B_{1} B_{2}-\left|D_{12}\right|^{2}$ for the s-ordering, $B_{1,2 s}=B_{1,2}+(1-s) / 2=\langle n\rangle_{\text {spont }} / 2+(1-s) / 2$, the mean number of spontaneous photons in the field $\langle n\rangle_{\text {spont }}$ equals in the pure case without losses and noise $2 \sinh ^{2}(g t)$; in this ideal case $K=-B=-B_{1}=-B_{2}$ (the negative value of this determinant reflects that the Glauber-Sudarshan quasi distribution never exists in the interaction), $\left|D_{12}\right|^{2}=B(B+1)$ and so $\left.K_{s}=-s\langle n\rangle_{\text {spont }} / 2 M+(1-s)^{2} / 4\right)$ in this case. Further the time-dependent intensity of the whole signal and idler field is $I_{j}=\left|\xi_{j}(t)\right|^{2}=M\left|\zeta_{j}(t)\right|^{2}, j=1,2$ and $L_{s}=$ $\left|\xi_{1}\right|^{2} B_{2 s}+\left|\xi_{2}\right|^{2} B_{1 s}-\left(\xi_{1} \xi_{2} D_{12}^{*}+\right.$ c.c. $), \xi_{j}=\sqrt{M} \zeta_{j}$. In the interaction picture we have for the time-dependent complex amplitudes

$$
\begin{aligned}
& \zeta_{1}(t)=\exp (-\gamma t / 2)\left[\zeta_{1}(0) u(t)+i \zeta_{2}^{*}(0) v(t) \exp (i \phi)\right],(2) \\
& \zeta_{2}(t)=\exp (-\gamma t / 2)\left[\zeta_{2}(0) u(t)+i \zeta_{1}^{*}(0) v(t) \exp (i \phi)\right] .
\end{aligned}
$$

Here $\gamma$ is the damping constant, $\zeta_{1,2}(0)$ are initial signal and idler complex stimulating amplitudes, $\phi$ is the pump- 
ing phase, $u(t)=\cosh (g t), v(t)=\sinh (g t), g$ and $t$ being the coupling constant proportional to quadratic susceptibility including the real pumping amplitude and interaction time, respectively. Further in the ideal case the quantum noise functions are $B_{1}=\left\langle\Delta \hat{a}_{1}^{\dagger} \Delta \hat{a}_{1}\right\rangle=B_{2}=\left\langle\Delta \hat{a}_{2}^{\dagger} \Delta \hat{a}_{2}\right\rangle=$ $B=|v|^{2}=\sinh ^{2}(g t), D_{12}=\left\langle\Delta \hat{a}_{1} \Delta \hat{a}_{2}\right\rangle=i \exp (i \phi) u v=$ $(i / 2) \sinh (2 g t) \exp (i \phi)$. The time-dependent noise functions $B_{1}=B_{2}=B$ and $D_{12}$ including losses and noise were given in [28] (equations (10.14a), [29] and their simplified form can be written in the interaction picture in the following way [30]:

$$
\begin{aligned}
B & =\frac{1}{2}\left(a_{1}+a_{3}\right)-1, D_{12}=\frac{i}{2} \exp (i \phi)\left(a_{3}-a_{1}\right), \\
K & =a_{1} a_{3}-a_{1}-a_{3}+1, \\
a_{1} & =\frac{\kappa_{1}}{\kappa_{5}}\left[1-\exp \left(-\kappa_{5} t\right)\right]+\exp \left(-\kappa_{5} t\right), \\
a_{3} & =\frac{\kappa_{3}}{\kappa_{6}}\left[1-\exp \left(\kappa_{6} t\right)\right]+\exp \left(\kappa_{6} t\right), \\
\kappa_{1,3} & =g \pm \gamma\left(\left\langle n_{\mathrm{d}}\right\rangle+1\right), \kappa_{5,6}=2 g \pm \gamma,
\end{aligned}
$$

where $\left\langle n_{\mathrm{d}}\right\rangle$ is the mean number of reservoir oscillators in a mode (for $\gamma=\left\langle n_{\mathrm{d}}\right\rangle=0$ we obtain the above values $B=$ $\left.\sinh ^{2}(g t), D_{12}=(i / 2) \sinh (2 g t) \exp (i \phi)\right)$. From these values including damping and losses it can be verified that $-B_{S} \leq K_{s}$ and in particular $-B \leq K$ for $s=1(K+B \geq 0$, i.e. a quantum Schwarz inequality $B+B^{2} \geq\left|D_{12}\right|^{2}$ holds, giving noise component in the joint photon-number distribution Eq.(7); this ensures its non-negativity) and the equality $K=-B$ occurs in the pure process without damping and losses only.

Hence we assume the flat spectrum of noise of photon pairs as an approximation. The number $M$ of degrees of freedom of photon pairs is a free parameter assumed to be determined from the second moments of experimental data. For signal or idler modes ( $\lambda_{2}$ or $\lambda_{1}$ equals zero) we have the standard superposition of signal and noise [28] (Sec. 5.3) and for the spontaneous process the signal or idler modes are described by the Mandel-Rice formula for the photon-number distribution and by the Rayleigh (Gamma) distribution for the integrated intensity.

For $s=1$ in Eq.(1) we obtain the normal generating function for the joint photon-number distribution $p\left(n_{1}, n_{2}\right)$, which is derived through the derivatives around $\lambda_{1}=\lambda_{2}=1$ in the standard way. Putting $\lambda_{1,2} \rightarrow-i s_{1,2}$ in terms of new Fourier variables $s_{1,2}$, the Fourier transforms with respect to $s_{1,2}$ provide the $s$-ordered joint integrated-intensity distribution $P_{S}\left(W_{1}, W_{2}\right), W_{1}=\left|\alpha_{1}\right|^{2}, W_{2}=\left|\alpha_{2}\right|^{2}, \alpha_{1,2}$ being the complex amplitudes of the signal and idler fields, respectively. The generating function $G_{s}(\lambda, \lambda)$ is then appropriate for obtaining quantities for the compound mode Eqs.(1,2), such as the sordered moments for the whole field, the integrated-intensity distribution $P_{S}(W), W=W_{1}+W_{2}$ related to s-ordering for the whole field, and if $s=1$ the photon-number distribution $p(n)$, $n=n_{1}+n_{2}$ and its factorial moments are obtained through derivatives around $\lambda=1$ and 0 , respectively. However, all these results were derived earlier (see review in [28], Sec. 10.2).

The corresponding first- and second-order moments are [27]

$$
\begin{aligned}
\left\langle W_{j}\right\rangle_{s} & =M B_{j s}+\left|\xi_{j}\right|^{2}, j=1,2, \\
\left\langle\left(\Delta W_{j}\right)^{2}\right\rangle_{s} & =M B_{j s}^{2}+2 B_{j s}\left|\xi_{j}\right|^{2}, j=1,2,
\end{aligned}
$$

$$
\left\langle\Delta W_{1} \Delta W_{2}\right\rangle_{s}=M\left|D_{12}\right|^{2}+\left(\xi_{1} \xi_{2} D_{12}^{*}+\text { c.c. }\right) .
$$

These expressions make it possible to determine also the noise functions $B_{j},\left|D_{12}\right|$ and the number of degrees of freedom $M$ of photon pairs from experimental data [27].

When we put $\zeta_{1}=\zeta_{2}=0$, we obtain the case of the spontaneous process, as discussed in [6]. In this case the joint integrated-intensity distribution is obtained by means of the Fourier transformation [6]

$$
\begin{aligned}
P_{S}^{\text {spont }}\left(W_{1}, W_{2}\right)= & \frac{1}{\Gamma(M)}\left(\frac{1}{K_{s}}\right)^{M}\left(\frac{K_{s}^{2} W_{1} W_{2}}{\left|D_{12}\right|^{2}}\right)^{(M-1) / 2} \\
& \times \exp \left[-\frac{\left(B_{2 s} W_{1} / B_{1 s}+W_{2}\right) B_{1 s}}{K_{s}}\right] \\
& \times I_{M-1}\left(2\left[\frac{\left|D_{12}\right|^{2} W_{1} W_{2}}{K_{s}^{2}}\right]^{1 / 2}\right), K_{s}>0
\end{aligned}
$$

where $I_{M}$ is the modified Bessel function and

$$
\begin{aligned}
& P_{s}^{\text {spont }}\left(W_{1}, W_{2}\right) \approx \frac{\left(W_{1} W_{2}\right)^{(M-1) / 2}}{\pi \Gamma(M) B_{1 s}^{M}} \\
& \quad \times \exp \left(-\frac{W_{1}+W_{2}}{2 B_{1 s}}\right) \frac{\sin A\left(W_{1}-W_{2}\right)}{W_{1}-W_{2}}, K_{s}<0,
\end{aligned}
$$

$A=\left(-K_{s} B_{1 s} / B_{2 s}\right)^{-1 / 2}$. The corresponding joint photonnumber distribution is [6]

$$
\begin{gathered}
p^{\text {spont }}(k, l)=\frac{1}{\Gamma(M)} \frac{(-K)^{l}\left(B_{1}+K\right)^{k-l}}{\left(1+B_{1}+B_{2}+K\right)^{k+M}} \\
\quad \times \sum_{r=\max (0, l-k)}^{l} \frac{\Gamma(k+M+r)}{r !(l-r) !(k-l+r) !} \frac{\left[\left(B_{1}+K\right)\left(B_{2}+K\right)\right]^{r}}{(-K)^{r}\left(1+B_{1}+B_{2}+K\right)^{r}} .
\end{gathered}
$$

For the noise component $B+K=0\left(B_{1}=B_{2}=B\right)$ we obtain the diagonal Mandel-Rice formula for photon pairs. The quality of the integrated-intensity distributions related to $s$ ordering is determined by the determinant $K_{s}[6] ; K_{s}=0$ gives a border between classical $\left(K_{S}>0\right.$, regular non-negative distributions) and quantum behaviour $\left(K_{s}<0\right.$, quasi distributions possessing negative values) and determines a relation between physical quantities, because it must be $B_{S}-\left|D_{12}\right|=$ $B+(1-s) / 2-\left|D_{12}\right|=0\left(B_{1}=B_{2}=B\right)$ and consequently we have for the threshold value of the ordering parameter $s_{\text {th }}=\langle n\rangle_{\text {spont }} / M+1-2\left|D_{12}\right|$. The quantum regime of oscillations in $P_{S}\left(W_{1}, W_{2}\right)$ [6] exhibiting phase-space interference [31] occurring for $K_{s}<0$ is just in coincidence with the quantum region of non-existence of $P_{S}(W)$ as a regular distribution (negative values) determined by $B_{s}-\left|D_{12}\right|<0$ (subPoissonian photon-number distribution $p(n)$ and its oscillations occur in correspondence to $s=1)$; in the opposite case when $K_{s}>0\left(B_{S}-\left|D_{12}\right|>0\right)$ the distribution $P_{S}\left(W_{1}, W_{2}\right)$ is a non-negative regular distribution and as well $P_{S}(W)$ exists as a regular non-negative distribution.

In principle one can expand the exponential function in the joint generating function to obtain multiple-sum expressions for the joint quantities through derivatives or Fourier transformation. However, they are cumbersome and impractical and for a sufficiently accurate description of the experimental data an approximation can be done. In this approximation we neglect interference of noises in both the signal and idler modes with the stimulated coherent components $\left(L_{S} \approx 0, D_{s} \approx 1\right.$ in 
the exponential function in Eq.(1)), having [27]

$$
\begin{aligned}
& P_{s}^{\text {stim }}\left(W_{1}, W_{2}\right)=\frac{1}{(2 \pi)^{2}} \int_{-\infty}^{\infty} \int_{-\infty}^{\infty} \frac{1}{D_{s}^{M}} \\
& \times \exp \left[-i s_{1}\left(W_{1}-I_{1}\right)-i s_{2}\left(W_{2}-I_{2}\right)\right] d s_{1} d s_{2} \\
&=P_{s}^{\text {spont }}\left(W_{1}-I_{1}, W_{2}-I_{2}\right), W_{1,2} \geq I_{1,2}
\end{aligned}
$$

which means that in this case all results discussed for the spontaneous process in [6] are valid around the stimulating intensity values $I_{1}, I_{2}$. One of the stimulating intensities can be zero. The corresponding joint photon-number distribution is [27]

$$
p^{\text {stim }}(k, l)=\sum_{k^{\prime}=0}^{k} \sum_{l^{\prime}=0}^{l} p^{\text {spont }}\left(k-k^{\prime}, l-l^{\prime}\right) \frac{I_{1}^{k^{\prime}} I_{2}^{l^{\prime}}}{k^{\prime} ! l^{\prime} !} \exp \left(-I_{1}-I_{2}\right) \text {. }
$$

These approximations are in general exact in the first moments, more accurate than $1 \%$ in the second moments, $3 \%$ in the third moments, $6 \%$ in the fourth moments and $10 \%$ in the fifth moments. For experimental data these approximations are still more accurate, which is given by values of $K_{s}$ close to zero and by larger number of degrees of freedom $M$ related to the spontaneous process (so that in the generating function (1) again $L_{S} \approx 0, D_{S} \approx 1$ in the exponential function and $D_{s}^{M} \approx\left(1+\lambda_{1} B_{1 s}+\lambda_{2} B_{2 s}\right)^{M}$ for $K_{s}<0$; in the opposite case the exact spontaneous joint distribution Eq.(5) is appropriate) [27]. If the stimulated components are quite dominant, the joint photon statistics are Poissonian,

$$
p^{\text {stim }}(k, l)=\frac{I_{1}^{k}}{k !} \exp \left(-I_{1}\right) \frac{I_{2}^{l}}{l !} \exp \left(-I_{2}\right)
$$

One can conclude in general that the most pronounced quantum effects are exhibited in the spontaneous process whereas the stimulated components mask them.

\section{ILLUSTRATIONSAND DISCUS- SIO N}

We can provide some illustrations of the above results for the stimulated process choosing the initial amplitudes $\left|\zeta_{1}(0)\right|=$ $\left|\zeta_{2}(0)\right|=1$ under the initial phase condition $\phi_{1}+\phi_{2}-\phi=$ $-\pi / 2$ giving the maximum non classical effects, $\phi_{1,2}$ being the phases of $\zeta_{1,2}(0)$. In

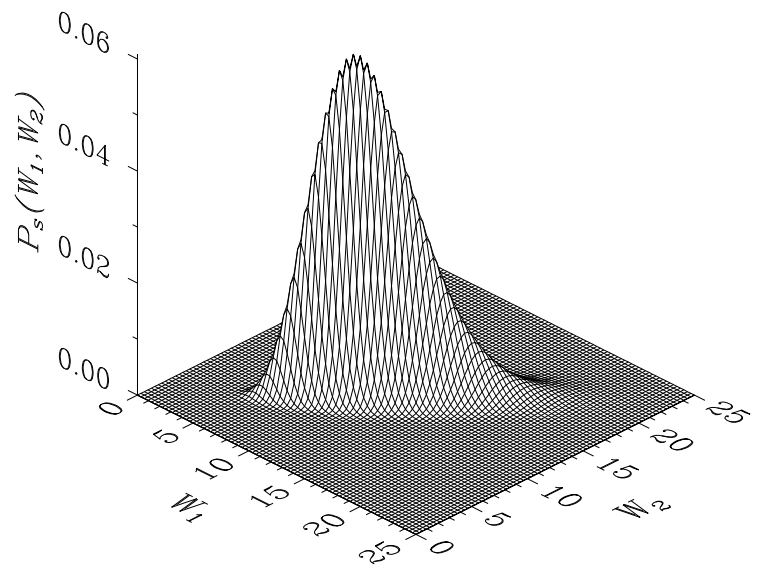

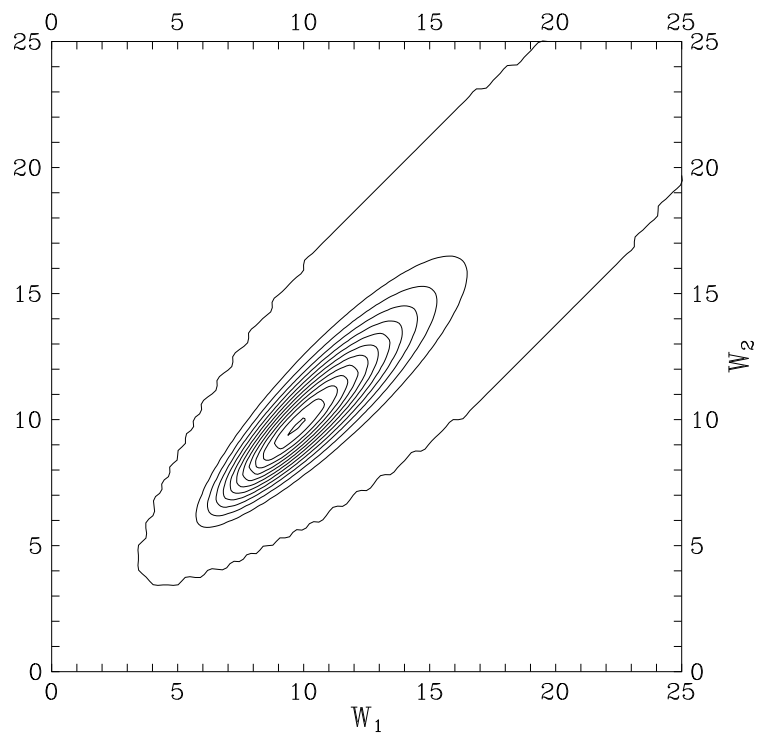

FIG. 1 Joint integrated-intensity distribution for $s=0.3, g t=0.6, M=10, \gamma / g=$ $0.1,\left\langle n_{\mathrm{d}}\right\rangle=1,\left|\zeta_{1}(0)\right|=\left|\zeta_{2}(0)\right|=1, \phi_{1}+\phi_{2}-\phi=-\pi / 2$ (a) (top) and its cut curves (b) (bottom).

Figure 1 we give the joint integrated-intensity distribution and its cut curves based on Eqs.(5) and (8) for $s=0.3, g t=$ $0.6, M=10, \gamma / g=0.1,\left\langle n_{\mathrm{d}}\right\rangle=0.5$ when $K_{s}=0.0680>0$, whereas in Figures 2 the same quantities are given for the same values of the parameters but $g t=0.9$ when $K_{s}=$ $-0.0411<0$, which are based on Eqs.(6) and (8). We see the change of quality
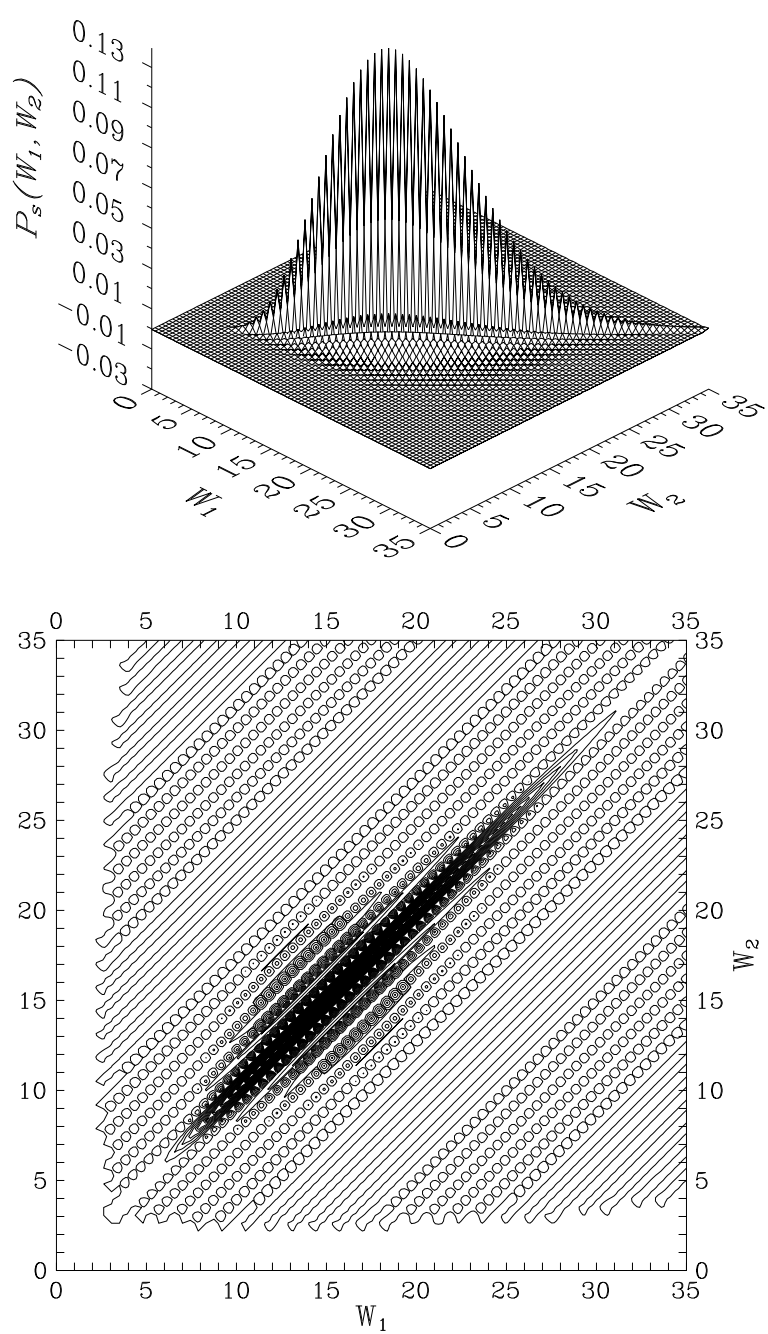

FIG. 2 As in Figures 1 but for $g t=0.9$. 
of the distribution from its regular non-negative behaviour to its quantum oscillating behaviour with negative values. In general with increasing $g t$ the values of $\left|K_{s}\right|$ increase and the frequency of oscillations $A$ decreases. In Figures 3 we give the stimulated joint photon-number distribution Eq.(9) where (7) has been used, together with its cut curves for the same values of parameters as in Figures 2 ( $s=1$ is appropriate). In the pure case without losses and noise $(K=-B)$, this distribution is diagonal. This is also reached in the limit $g t \rightarrow \infty$ provided that the nonlinearity is stronger than damping $(g>\gamma)$, because in this limit the influence of losses and noise relatively successively decreases (see Eq.(3)) and also the argument of the exponential function in Eq.(1) tends to zero in this case under the above phase condition.
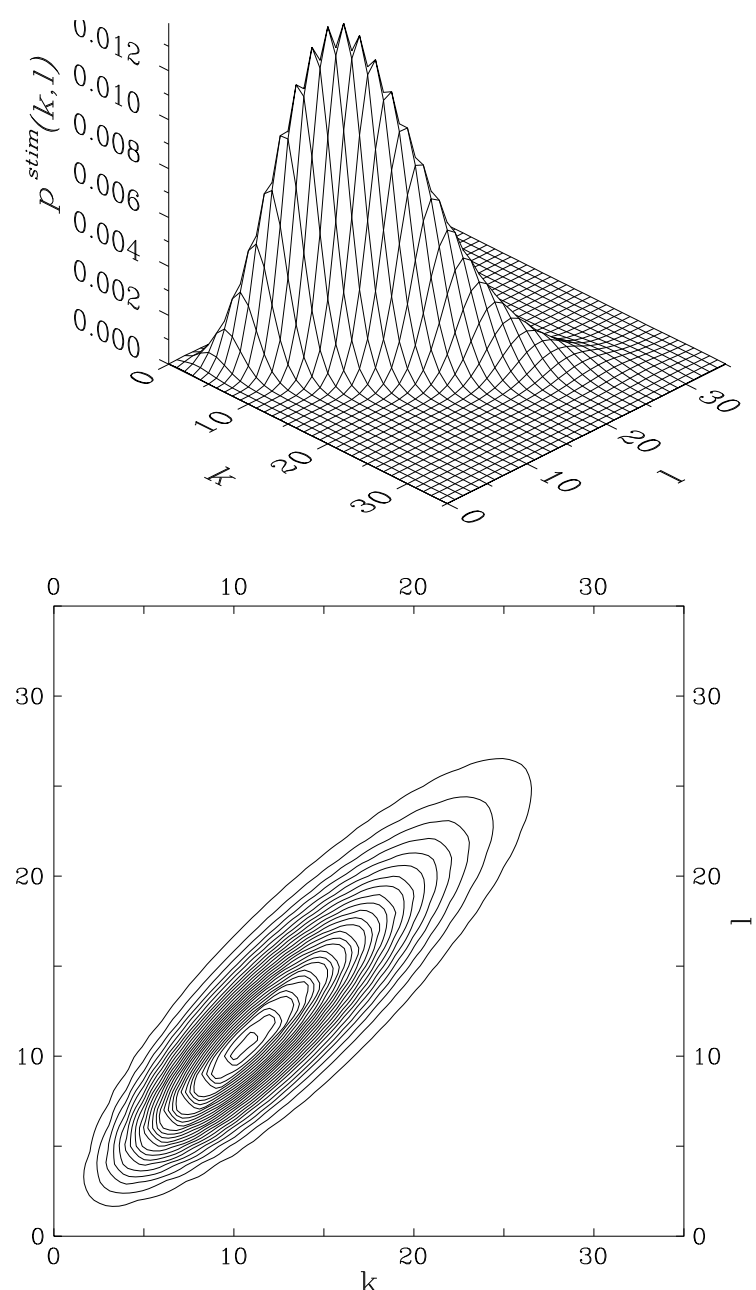

FIG. 3 Joint photon-number distribution for the same values of parameters as in Figures 2 ( $s=1$ is appropriate) (a) (top) and its cut curves (b) (bottom).

\section{C O N C L U S I O N}

In this paper we have reviewed a description of the quantum statistical properties of the multi mode spontaneous and stimulated processes of down-conversion. We have exhibited expressions for joint distributions of photon numbers and integrated intensities in signal and idler fields of multimode parametric down-conversion, including the border between classical and quantum behaviour. The description can be applied for time evolution of the corresponding statistical quantities including losses, noise and stimulating fields or as a tool based on the experimental data for determination of all relevant physical quantities.

\section{Acknowledgement}

This work was supported by the Research Project "Measurement and Information in Optics" MSM 6198959213, EU Project COST OCP11.003, Project 202/05/0498 of the Czech Grant Agency and the Centre of Modern Optics LC06007 of the Czech Ministry of Education.

\section{References}

[1] D.F. Walls, G.J. Milburn, Quantum Optics (Springer, Berlin, 1994) chap. 5.

[2] L. Mandel, E. Wolf, Optical Coherence and Quantum Optics ( Cambridge Univ., Cambridge, 1995) chap. 22.4.

[3] J. Peřina, Z. Hradil, B. Jurčo:, Quantum Optics and Fundamentals of Physics (Kluwer, Dordrecht, 1994) chap. 8.

[4] 0. Haderka, J. Peřina Jr., M. Hamar, J. Peřina, Phys. Rev. A 71, 033815 (2005).

[5] J. Peřina Jr., 0. Haderka, M. Hamar, "Statistical properties of twin beams generated in spontaneous parametric down-conversion", arXiv: quant-ph/0310065.

[6] J. Peřina, J. Křepelka, J. Opt. B: Quant. Semiclass. Opt. 7246 (2005).

[7] G. Di Giuseppe, M. Atature, M.D. Shaw, A.V. Sergienko, B.E.A. Saleh, M.C. Teich, A.J. Miller, S.W. Nam, J. Martinis, Phys. Rev. A 68063817 (2003).

[8] M.J. Fitch, B.C. Jacobs, T.B. Pittman, J.D. Franson, Phys. Rev. A 68 043814 (2003).

[9] Y. Zhang, K. Kasai, M. Watanabe, Opt. Lett. 271244 (2002).

[10] M. Vasilyev, S.-K. Choi, P. Kumar, G.M. D’Ariano, Phys. Rev. Lett. 84235 (2000).

[11] E. Waks, E. Diamanti, B.C. Sanders, S.D. Barlett, Y. Yamamoto, Phys. Rev. Lett. 92113602 (2004).

[12] S. Feng, 0. Pfister, Phys. Rev. Lett. 92203601 (2004).

[13] A.I. Lvovsky, H. Hansen, T. Aichele, 0. Benson, J. Mlynek, S. Schiller, Phys. Rev. Lett. 87050402 (2001).

[14] B.M. Jost, A.V. Sergienko, A.F. Abouraddy, B.E.A. Saleh, M.C. Teich, Opt. Express 381 (1998).

[15] E. Brambilla, A. Gatti, M. Bache, L.A. Lugiato, Phys. Rev. A 69023802 (2004).

[16] G. Brida, M. Genovese, M. Gramegna, M.L. Rastello, M. Chekhova, L. Krivitsky, J. Opt. Soc. Am. B 22488 (2005).

[17] Y.-H. Kim, W.P. Grice, J. Opt. Soc. Am. B 22493 (2005).

[18] J.A.O. Huguenin, M.P. Almeida, P.H.S. Ribeiro, A.Z. Khoury, Phys. Rev. A 71043818 (2005).

[19] Y. Zhang, K. Hayasaka, K. Kasai, Phys. Rev. A 71062341 (2005).

[20] L. Neves, G. Lima, J.G.A. Gómez, C.H. Monken, C. Saavedra, S. Pádua, Phys. Rev. Lett. 94100501 (2005).

[21] K.J. Resch, J.S. Lundeen, A.M. Steinberg, Phys. Rev. Lett. 88113601 (2002).

[22] K.J. Resch, J.S. Lundeen, A.M. Steinberg, J. Mod. Opt. 49487 (2002). 
[23] K.J. Resch, J.S. Lundeen, A.M. Steinberg, Phys. Rev. A 63020102 (R) (2001).

[24] K.J. Resch, J.S. Lundeen, A.M. Steinberg, Phys. Rev. Lett. 87123603 (2001).

[25] K.J. Resch, P. Walther, A. Zeilinger, Phys. Rev. Lett. 94070402 (2005).

[26] J. Fan, A. Dogariu, L.J. Wang, Opt. Lett. 301530 (2005).
[27] J. Peřina, J. Křepelka, Opt. Commun. (2006) in print.

[28] J. Peřina, Quantum Statistics of Linear and Nonlinear Optical Phenomena (Kluwer, Dordrecht, 1991).

[29] L. Mišta, J. Peřina, Czech. J. Phys. B 28392 (1978).

[30] A. Mebrahtu, J. Mod. Opt. 52813 (2005).

[31] W.P. Schleich, Quantum Optics in Phase Space (Wiley-VCH, Berlin, 2001), chaps. $4,7,8$. 